\title{
AVALIAÇÃO DA POTENCIALIDADE DE PROCESSOS PSEUDO-FENTON PARA REMEDIAÇÃO DE SOLOS CONTAMINADOS POR DIESEL
}

\author{
Carolina Acioli Pereira e Mônica Regina da Costa Marques* \\ Instituto de Química, Universidade do Estado do Rio de Janeiro, Rua São Francisco Xavier, 524, 20550-900 Rio de Janeiro - RJ, \\ Brasil \\ Daniel Vidal Pérez \\ EMBRAPA Solos, Rua Jardim Botânico, 1024, 22460-000 Rio de Janeiro - RJ, Brasil
}

Recebido em 16/9/08; aceito em 15/4/09; publicado na web em 17/9/09

\begin{abstract}
EVALUATION OF POTENCIAL FENTON-LIKE PROCESS TO THE REMEDIATION OF CONTAMINATED SOILS BY DIESEL. The use of the Fenton's reagent process has been investigated for the remediation of a Brazilian soil contaminated by diesel. Laboratory experiments were conducted in batch experiments. Slurries, consisting of $10 \mathrm{~g}$ of diesel-contaminated soil and 30 $\mathrm{mL}$ of Fenton's Reagent $\left(0.41 \mathrm{~mol} \mathrm{~L}^{-1} \mathrm{H}_{2} \mathrm{O}_{2}\right.$ and $\left.0.18 \mathrm{~mol} \mathrm{~L}^{-1} \mathrm{FeSO}_{4}\right)$. The experiments were monitored during 24,48 and $72 \mathrm{~h}$. The efficiency of the Fenton treatment was dependent on the time of contact between soil and Fenton's reagents and matrix characteristics, probably iron content. Data suggested that no iron addition is needed for the application of Fenton-like treatment for the remediation of diesel-contaminated iron rich soils after $72 \mathrm{~h}$ reaction.
\end{abstract}

Keywords: remediation; Fenton's reagent; soil.

\section{INTRODUÇÃO}

A contaminação por hidrocarbonetos tem-se tornado uma das grandes preocupações ambientais, uma vez que este tipo de substância xenobiótica interfere no ecossistema da área afetada, poluindo solo, ar, fauna, vegetação, águas superficiais e subterrâneas. ${ }^{1}$ A quantidade de resíduos industriais contaminados com esses poluentes, conhecidos como recalcitrantes, tem aumentado significativamente ao longo dessas últimas décadas, por práticas inadequadas de disposição de resíduos químicos, que levam, muitas das vezes, a vazamento durante seu manuseio, transporte ou armazenamento. ${ }^{2}$ Muitos dessas substâncias possuem um alto risco para a saúde humana e, por vezes, até mesmo aos próprios micro-organismos que, eventualmente, poderiam vir a fazer a sua degradação.

Atualmente, muitas pesquisas vêm sendo desenvolvidas visando a remediação de solos contaminados com poluentes orgânicos..$^{3-5}$ Dentre estas técnicas, os processos químicos definidos como Processos Oxidativos Avançados (POA) constituem tecnologias alternativas para o tratamento de vários contaminantes orgânicos recalcitrantes e têm sido aplicados, com sucesso, na remediação in situ de solos contaminados. Estes tratamentos se baseiam na formação de radicais hidroxilas $\left(\mathrm{OH}^{\circ}\right)$ altamente oxidantes $\left(\mathrm{E}^{\circ}=2.08 \mathrm{~V}\right)$, capazes de reagir com praticamente todas as classes de compostos orgânicos e inorgânicos. ${ }^{6,7} \mathrm{~A}$ grande vantagem desses processos está no fato de que, na maioria dos casos, os produtos de degradação final não são poluentes ao ambiente. ${ }^{8}$ A oxidação química usando o reagente de Fenton tem se mostrado uma técnica promissora para esses casos, pois, na sua concepção, pode levar vários contaminantes orgânicos à completa mineralização ou a compostos que sejam inócuos ou biodegradáveis no meio ambiente. ${ }^{9}$

$\mathrm{O}$ termo reagente de Fenton refere-se a um processo redox, que consiste em uma mistura aquosa de Fe (II) e peróxido de hidrogênio onde ocorre transferência de elétrons entre $\mathrm{o}_{2} \mathrm{O}_{2}$ e o redutor. Assim, quando o peróxido de hidrogênio é usado para degradar contaminantes em solos, que apresentam naturalmente altos teores de ferro,

*e-mail: monica@pesquisador.cnpq.br que agirão, então, como catalisadores da reação, esse processo é denominado de tipo-Fenton. ${ }^{10}$

A reação 1, sendo reconhecida como a reação predominante do processo, mostra como é gerado o radical hidroxila. ${ }^{11}$

$\mathrm{Fe}^{2+}+\mathrm{H}_{2} \mathrm{O}_{2} \rightarrow \mathrm{Fe}^{3+}+\mathrm{OH}^{\cdot}+\mathrm{OH}^{-}$

Nas reações posteriores as reações 2 e 3, notam-se a regeneração do íon ferroso a partir do íon férrico que reage com o peróxido de hidrogênio e com o radical hidroperoxil $\left({ }^{\circ} \mathrm{O}_{2} \mathrm{H}\right)$, dando sustentabilidade ao processo de Fenton. ${ }^{12}$

$\mathrm{Fe}^{2+}+\mathrm{HO}^{\bullet} \rightarrow \mathrm{Fe}^{3+}+\mathrm{HO}^{\bullet}$

$\mathrm{Fe}^{3+}+\mathrm{H}_{2} \mathrm{O}_{2} \leftrightarrow \mathrm{Fe}-\mathrm{OOH}_{2}^{+}+\mathrm{H}^{+}$

$\mathrm{Fe}-\mathrm{OOH}_{2}^{+} \rightarrow \mathrm{HO}_{2}^{\cdot}+\mathrm{Fe}^{2+}$

$\mathrm{Fe}_{2+}+\mathrm{HO}_{2} \cdot \rightarrow \mathrm{Fe}^{3+}+\mathrm{HO}_{2}^{-}$

$\mathrm{Fe}^{3+}+\mathrm{HO}_{2}^{\cdot} \rightarrow \mathrm{Fe}^{2+}+\mathrm{H}^{+}+\mathrm{O}_{2}$

$\mathrm{H}_{2} \mathrm{O}_{2}+\mathrm{HO}^{\bullet} \rightarrow{ }^{\circ} \mathrm{O}_{2} \mathrm{H}+\mathrm{H}_{2} \mathrm{O}$

O mecanismo de oxidação de hidrocarbonetos catalisado por radicais hidroxila (Equações 8 a 11), provado e aceito desde 1971, mostra a oxidação de compostos orgânicos $(\mathrm{RH})$ pelo radical hidroxila ( $\mathrm{HO}^{*}$ ), formando radicais $\mathrm{R}^{*}$ os quais são altamente reativos e podem também ser oxidados.

$$
\begin{aligned}
& \mathrm{R}-\mathrm{H}+\mathrm{HO}^{\bullet} \rightarrow \mathrm{R}^{\bullet}+\mathrm{H}_{2} \mathrm{O} \\
& \mathrm{R}^{\bullet}+\mathrm{H}_{2} \mathrm{O}_{2} \rightarrow \mathrm{ROH}+\mathrm{HO}^{\bullet} \\
& \mathrm{R}^{\bullet}+\mathrm{OH}^{\bullet} \rightarrow \mathrm{ROH} \\
& 2 \mathrm{R}^{\bullet} \rightarrow \text { Produtos }
\end{aligned}
$$


Estudos cinéticos mostraram que a reação está condicionada tanto pelo tipo de substrato orgânico presente na matriz do solo, quanto pelas condições em que se realiza a oxidação. No entanto, é consensual que o $\mathrm{pH}$ e as concentrações de peróxido de hidrogênio e de ferro influenciam a velocidade da reação. ${ }^{13-15}$

O território brasileiro caracteriza-se por uma grande diversidade de tipos de solos. Destes, os Latossolos ocupam 38,7\% da área total do país, distribuindo-se em quase todo território nacional. ${ }^{16}$ Uma de suas características mais importantes e que, por isso, também vem sendo usada para sua diferenciação no sistema brasileiro de classificação de solo, ${ }^{17}$ é o teor de ferro.

A oxidação com o reagente de Fenton está diretamente ligada à concentração de íons Fe no meio reacional. ${ }^{18}$ Alguns trabalhos relatam a adição de sais de ferro dissolvidos ou de ferro mineral (por exemplo, goethita e magnetita) ao solo a ser tratado. Entretanto, estes estudos são limitados a solos areonosos como, por exemplo, o recente trabalho de Silva et al.. ${ }^{19}$ Neste trabalho, os autores avaliaram a degradação de hidrocarbonetos policíclicos aromáticos (HPAs) presentes no óleo diesel comercial em dois tipos de solos arenosos com diferentes teores de ferro endógeno ( $\mathrm{S} 1=9,1$ e S2 = 0,6 Fe $/ \mathrm{g} \mathrm{kg}^{-1}$ ). Neste caso, o processo pseudofotofenton pode ser usado tratar áreas contaminadas por HPAs apresentando um nível satisfatório de degradação (acima de 80\%) obtido em condições econômicas (ferro endógeno, baixa concentração de peróxido, curto tempo de exposição à radiação e sem ajuste de $\mathrm{pH}$ do solo). Entretanto, até o momento, não foram descritos trabalhos envolvendo o uso do reagente de Fenton em solos argilosos com elevado teor natural de ferro. Assim, para se avaliar a influencia do ferro natural normalmente presente nos solos brasileiros, no presente trabalho pretende-se avaliar a potencialidade do processo pseudoFenton na remediação de um solo argiloso rico em ferro contaminado com óleo diesel.

\section{METODOLOGIA}

O horizonte superficial $(0-15 \mathrm{~cm})$ de um Latossolo Vermelho (LV) do município de Lagoa Formosa (MG) foi coletado pela EMBRAPA Solos. Antes da contaminação, as propriedades físicas e químicas (Tabela 1) foram determinadas conforme Embrapa. ${ }^{20} \mathrm{~A}$ fração argila é dominada por óxido de alumínio (gibbsita - 53\%), seguida do alumínio-silicato tipo caolinita (34\%) e dos óxidos de ferro (goetita - $8 \%$ e hematita - 5\%). O teor de ferro presente na fração argila foi determinado após ataque ácido seguido de titulação com EDTA

Tabela 1. Propriedades físicas e químicas dos solos antes da contaminação

\begin{tabular}{lc}
\hline Propriedades & LV \\
\hline Areia (\%) & 17 \\
Silte $(\%)$ & 23 \\
Argila (\%) & 60 \\
$\mathrm{pH}$ & 5,5 \\
$\mathrm{C}$ orgânico, g kg & \\
$\mathrm{N}$ total, $\mathrm{g} \mathrm{kg}^{-1}$ & 3,06 \\
Fe total $\left(\mathrm{g} \mathrm{kg}^{-1}\right)$ & 0,12 \\
\hline
\end{tabular}

Pela análise cromatográfica do óleo diesel, foram observados picos com tempos de retenção entre 5 e 40 min, com um perfil de série homóloga de hidrocarbonetos de cadeia alifática, constituído por cadeias carbônicas contendo de 9 a 26 átomos de carbono (pureza acima de $99 \%$ ). O teor de cada hidrocarboneto alifático no óleo diesel bruto (Tabela 2) foi determinado a partir da curva de calibração do padrão tetradecano.
A contaminação dos solos foi feita a partir do óleo diesel dissolvido em n-pentano e aplicado em cada solo, para se obter, então, uma concentração final de $50 \mathrm{~g} \mathrm{~kg}^{-1}$. Os solos contaminados foram armazenados à temperatura ambiente por, no mínimo, 15 dias. Depois disso, foi testada a recuperação de óleo diesel, obtendo-se uma média de $99 \%$ de recuperação.

Para testes com o reagente de Fenton, em $10 \mathrm{~g}$ de solo contaminado, foi adicionado o reagente de Fenton $\left(\mathrm{H}_{2} \mathrm{O}_{2} 30 \%\right.$ e solução ácida de $\mathrm{FeSO}_{4} 0,18 \mathrm{~mol} \mathrm{~L}^{-1}$ ) e ajustado o $\mathrm{pH}$ a 3 com gotas de solução de $\mathrm{NaOH}(1 \mathrm{~mol} / \mathrm{L})$. Após o tempo estipulado para remediação, o solo foi separado por centrifugação (2.500 rpm) e o teor de hidrocarboneto residual extraído com solvente hexano por $24 \mathrm{~h} \cdot{ }^{21}$ Após evaporação do solvente, o resíduo foi seco, até peso constante em balança analítica de precisão de $0,00001 \mathrm{~g}$ (considerou-se que o resíduo do balão é basicamente o peso de hidrocarbonetos não reagido para o cálculo do rendimento bruto - método gravimétrico) e o teor de hidrocarbonetos alifáticos quantificado por cromatografia gasosa de alta resolução (CGAR) sob as seguintes condições de análise: Coluna capilar HP$5 \mathrm{MS}(30 \mathrm{~m} \times 0,32 \mathrm{~mm}, 0,25 \mu \mathrm{m})$, temperatura inicial de $100^{\circ} \mathrm{C}$, com rampa de programação de $5^{\circ} \mathrm{C} / \mathrm{min}$, e temperatura final $300^{\circ} \mathrm{C}$. Os testes foram feitos em triplicata e o desvio padrão foi de $5 \%$. O resíduo foi analisado por espectrofotometria na região do infravermelho com transformada de Fourier (FTIR) em pastilha de aproximadamente $0,5 \%$ de amostra em $\mathrm{KBr}$, com resolução de $4 \mathrm{~cm}^{-1}$.

Tabela 2. Teor dos hidrocarbonetos presentes no óleo diesel medido por cromatografia gasosa acoplada ao espectrômetro de massas

\begin{tabular}{lcc}
\hline Hidrocarboneto & $\mathrm{t}_{\mathrm{r}}(\mathrm{min})^{\mathrm{a}}$ & Teor no óleo diesel (\%) \\
\hline Nonano & 4,46 & 0,4 \\
Decano & 5,86 & 4,2 \\
Undecano & 8,04 & 12,3 \\
Dodecano & 10,80 & 15,0 \\
Tridecano & 13,76 & 17,1 \\
Tetradecano & 16,68 & 12,7 \\
Pentadecano & 19,45 & 10,5 \\
Hexadecano & 22,06 & 8,1 \\
Heptadecano & 24,52 & 5,6 \\
Octadecano & 26,85 & 4,6 \\
Nonodecano & 29,06 & 3,2 \\
Icosano & 31,17 & 2,2 \\
Henicosano & 33,18 & 1,4 \\
Docosano & 35,10 & 1,0 \\
Tricosano & 36,94 & 0,7 \\
Tetracosano & 38,71 & 0,5 \\
Pentacosano & 40,41 & 0,3 \\
Hidrocarbonetos totais & & 99,8 \\
(soma) & & \\
\hline & &
\end{tabular}

${ }^{\mathrm{a}} \mathrm{t}_{\mathrm{r}}$ : tempo de retenção

\section{RESULTADOS E DISCUSSÃO}

Os resultados obtidos dos testes de remediação estão apresentados na Tabela 3. Analisando comparativamente o rendimento bruto da remediação, para o tempo de reação de 24 h (Teste 1 e 4), pode concluir-se, primeiramente, que o melhor rendimento foi obtido pelo 
uso da solução ácida de Fe (II). Nesse sentido, os dados sugerem que a eficiência do reagente de Fenton está diretamente relacionada com a quantidade e/ou especiação de ferro no processo. Apesar do LV ser rico em ferro, este elemento está na forma de Fe (III) que precisa ser reduzido à $\mathrm{Fe}$ (II) que é o catalisador da reação de Fenton (reações 3 e 4). Em solos arenosos, ${ }^{22}$ a disponibilidade do ferro endógeno é maior, o que facilita a oxidação, já que não há a superfície reativa das argilas para interferir.

Novos testes foram conduzidos, nas mesmas condições de concentração de $\mathrm{H}_{2} \mathrm{O}_{2}$ e sem adição de $\mathrm{Fe}$ (II), porém aumentando-se o tempo de contato, visando aumentar a disponibilidade do Fe (II). Os resultados (testes 2 e 3 - Tabela 2), demonstraram que houve uma significativa influência do tempo de contato na remediação de solos contaminados, incrementando em $22 \%$ o rendimento da remediação para o tempo de contato de $72 \mathrm{~h}$.

Tabela 3. Condições reacionais de remediação com reagente de Fenton

\begin{tabular}{lccccc}
\hline Teste & Solo & Tempo (h) & \multicolumn{2}{c}{ Volume $(\mathrm{mL})$} & $\begin{array}{c}\text { Rendimento } \\
\text { médio (\%) }\end{array}$ \\
& & & $\mathrm{H}_{2} \mathrm{O}_{2}{ }^{\mathrm{a}}$ & $\mathrm{Fe}(\mathrm{II})^{\mathrm{b}}$ & \\
\hline 1 & LV & 24 & 25 & - & 60 \\
2 & LV & 48 & 25 & - & 72 \\
3 & LV & 72 & 25 & - & 82 \\
4 & LV & 24 & 25 & 5 & 76 \\
5 & LV & 48 & 25 & 5 & 78 \\
6 & LV & 72 & 25 & 5 & 84 \\
\hline
\end{tabular}

${ }^{\text {a }}$ Concentração da $\mathrm{H}_{2} \mathrm{O}_{2}$ de $0,41 \mathrm{~mol} \mathrm{~L}^{-1}$; ${ }^{\mathrm{b}}$ Concentração de ferro de $0,18 \mathrm{~mol} \mathrm{~L}^{-1} ;{ }^{\mathrm{c}}$ Rendimento médio (determinado pelo método gravimétrico).

Sendo assim, outros testes foram realizados com o intuito de verificar se haveria um aumento significativo da remediação com a adição da solução ácida de sulfato ferroso através da reação de Fenton. Os resultados (testes 5 e 6 - Tabela 2) mostram que o aumento do tempo de reação, de 24 a $72 \mathrm{~h}$, ocasionou um incremento médio de $8 \%$ no rendimento da remediação pela reação de Fenton. Contudo, quando comparados os resultados da remediação, após 72 h, com e sem a adição de $\mathrm{Fe}(\mathrm{II})$, observa-se que a diferença entre os tratamentos não foi significativa. Ou seja, em intervalos curtos, observa-se uma expressiva atividade catalítica do íon ferroso. Porém em intervalos longos, a remediação sem a adição de Fe(II) apresenta rendimentos similares àquela onde houve a adição desse elemento. Este resultado sugere, então, que os óxidos de ferro endógenos participam do processo de oxidação, não sendo necessária a adição externa de ferro. Além disso, do ponto de vista prático, observa-se que a adição do catalisador (solução ácida de íon ferroso) acarreta em uma reação altamente exotérmica, o que não ocorreu quando a remediação foi realizada no LV sem a adição da solução de Fe(II). Este fato demonstra a potencialidade em utilizar-se ferro mineral no lugar da solução de íon ferroso, uma vez que o aquecimento excessivo do solo pode causar a sua esterilização.

Por fim, o resultado do solo remediado nas condições do teste 6 sugere que não houve uma seletividade na remediação, ou seja, todos os hidrocarbonetos foram remediados com a mesma velocidade.
Pela análise dos extratos hexânico, observou-se o aparecimento das seguintes bandas: $1690 \mathrm{~cm}^{-1}$, característica de compostos carbonilados insaturados e $1715 \mathrm{~cm}^{-1}$, característica de carbonilas de cetonas/aldeídos saturados. Este resultado sugere que nas condições reacionais, ainda não foi possível a completa mineralização dos hidrocarbonetos alifáticos.

\section{CONCLUSÃO}

O resultado da remediação do Latossolo Vermelho (LV), um solo argiloso com elevado teor natural de óxido férrico, contaminado com óleo diesel, revelou-se eficiente para o processo de remediação do tipo Fenton, sem necessidade de adição de sais de ferro.

\section{AGRADECIMENTOS}

À FAPERJ, pela bolsa de estudos de C. A. Pereira, ao CNPq e à CAPES pelo apoio financeiro.

\section{REFERÊNCIAS}

1. Millioli, V. S.; Freire, D. D. C.; Cammarota, M. C.; J. Hazard. Mater. 2003, 79.

2. Kong, S.; Watts, R. J.; Choi, J.; Chemosphere 1998, 37, 1473.

3. Watts, R. J.; Haller, D. R.; Jones, A. P.; Tell, A. L.; J. Hazard. Mater. 2000, 76, 73 .

4. Quan, H. N.; Tell, A. L.; Watts, R. J.; J. Hazard. Mater. 2003, 102, 277.

5. Ferguson, S. H.; Woinarski, A. Z.; Snape, I.; Morris, C. E.; Revill, A. T.; Cold Regions Science and Technology 2004, 40, 47.

6. Zamora, P. P.; Tiburtius, E. R. L.; Emme, A.; Leal, E. S.; Quim. Nova 2005, 28, 61 .

7. Nogueira, R. F. P.; Trovó, A. G.; Silva, M. R. A.; Villa, R. D.; Quim. Nova 2007, 30, 400.

8. Watts, R. J.; Dilly, S. E.; J. Hazard. Mater. 1996, 51, 209.

9. Rivas, F. J.; J. Hazard. Mater. 2006, 138, 234.

10. Watts, R. J.; Stanton, P. C.; Waste Resour. 1998, 33, 1405.

11. Walling, C.; Acc. Chem Res. 1975, 8, 125.

12. Neyens, E.; Baeyens, J.; J. Hazard. Mater. 2003, 98, 33.

13. McBride, M. B.; Environmental Chemistry of Soil, Oxford University Press: New York, 1994.

14. Flotron, V.; Delteil, C.; Padellec, Y.; Camel, V.; Chemosphere 2004, 59. 1427 .

15. Kang, N.; Hua, I.; Chemosphere 2005, 23, 1561

16. Resende, M.; Curi, N.; Santana, D. P.; Pedologia e Fertilidade do solo: Interações e Aplicações, Potafos: Piracicaba, 1988.

17. EMBRAPA; Sistema Brasileiro de Classificação de Solos, 2a . ed., Embrapa Solos: Rio de Janeiro, 2006.

18. Tarr, M. A.; Degradation Methods for Wastes and Pollutants: Environmental and Industrial Applications, Marcel Dekker: New York, 2003, cap. 4.

19. Silva, P. T. S.; Locatelli, M. A. F.; Jardim, W. F.; Barros Neto, B.; Motta, M.; Castro, G. R.; Silva, V. L.; J. Braz. Chem. Soc. 2008, 19, 329.

20. EMBRAPA; Manual de Métodos de Análise de Solo, 2a. ed., Centro Nacional de Pesquisa de Solos: Rio de Janeiro, 1997.

21. Ferreira, M. M.; Fernandes, B.; Curi, N.; Revista Brasileira de Ciência do Solo 1999, 23.

22. Usepa; Método 3540 C, 1996.507. 